\title{
Performance monitoring of the Geumdang Bridge using a dense network of high-resolution wireless sensors
}

\author{
Jerome P Lynch ${ }^{1}$, Yang Wang ${ }^{2}$, Kenneth $\mathrm{J} \mathrm{Loh}^{1}$, Jin-Hak $\mathbf{Y i}^{3}$ and \\ Chung-Bang Yun ${ }^{4}$ \\ ${ }^{1}$ Department of Civil and Environmental Engineering, University of Michigan, Ann Arbor, \\ MI, USA \\ ${ }^{2}$ Department of Civil and Environmental Engineering, Stanford University, Stanford, CA, \\ USA \\ ${ }^{3}$ Coastal Engineering Research Department, Korea Ocean Research and Development \\ Institute, Ansan, Gyeonggi, Korea \\ ${ }^{4}$ Department of Civil and Environmental Engineering, Korea Advanced Institute of Science \\ and Technology, Daejeon, Republic of Korea \\ E-mail: jerlynch@umich.edu
}

Received 29 December 2005, in final form 8 August 2006

Published 3 October 2006

Online at stacks.iop.org/SMS/15/1561

\begin{abstract}
As researchers continue to explore wireless sensors for use in structural monitoring systems, validation of field performance must be done using actual civil structures. In this study, a network of low-cost wireless sensors was installed in the Geumdang Bridge, Korea to monitor the bridge response to truck loading. Such installations allow researchers to quantify the accuracy and robustness of wireless monitoring systems within the complex environment encountered in the field. In total, 14 wireless sensors were installed in the concrete box girder span of the Geumdang Bridge to record acceleration responses to forced vibrations introduced by a calibrated truck. In order to enhance the resolution of the capacitive accelerometers interfaced to the wireless sensors, a signal conditioning circuit that amplifies and filters low-level accelerometer outputs is proposed. The performance of the complete wireless monitoring system is compared to a commercial tethered monitoring system that was installed in parallel. The performance of the wireless monitoring system is shown to be comparable to that of the tethered counterpart. Computational resources (e.g. microcontrollers) coupled with each wireless sensor allow the sensor to estimate modal parameters of the bridge such as modal frequencies and operational displacement shapes. This form of distributed processing of measurement data by a network of wireless sensors represents a new data management paradigm associated with wireless structural monitoring.
\end{abstract}

(Some figures in this article are in colour only in the electronic version)

\section{Introduction}

Structural monitoring systems are widely adopted to record the response of large-scale civil structures subjected to extreme loadings and harsh environmental conditions. For example, the economic importance of long-span bridges often warrants the installation of structural monitoring systems with many bridges monitored world-wide including those in the United States [1], Japan [2], and China [3]. In recent years, engineers have begun to extend the functionality of structural monitoring systems so that they are capable of autonomous detection of the onset of structural damage. Termed a structural health 
monitoring (SHM) system, such systems will provide real-time safety assessment for facility owners and managers. However, the high costs often associated with the installation and upkeep of structural monitoring systems have retarded their widespread adoption. Specifically, commercial monitoring systems employ extensive lengths of coaxial wiring between sensors and a central data repository. Installation of wires in a structure can drive monitoring system costs high, often in excess of $\$ 5000$ per sensing channel [4].

To enhance the economic attractiveness of structural monitoring systems, new technologies are needed to reduce monitoring system costs. One likely means of reducing costs is the eradication of extensive lengths of coaxial wire needed between sensors and the data repository. In response to this need, the structural engineering community has begun to explore the use of wireless sensors in various structural monitoring applications [5]. Wireless sensors are defined by their use of wireless communication to transfer data from sensor to sensor and from sensor to data repository. In addition to wireless communications, wireless sensors also integrate analog-to-digital converters (ADCs) and low-power microcontrollers for their operation. To be considered a viable substitute for traditional tethered monitoring systems, wireless sensors must offer a level of performance on a par with existing systems. Some performance specifications required for structural monitoring include high ADC resolutions (16bit or higher) and far communication ranges (to allow internodal distances of over $100 \mathrm{~m}$ ). In addition, battery-powered wireless sensors installed in difficult to reach locations must be of sufficiently low power that their operational life spans are on the order of years. These specifications strongly influence which commercial off-the-shelf (COTS) components can be selected for the design of a wireless sensor prototype. To date, a number of academic and commercial prototypes meeting such requirements have been proposed and tested in the laboratory [6].

Although wireless sensor prototypes have been thoroughly tested in the laboratory environment, wireless monitoring systems have only begun to be tested in the complex field setting. For example, the Alamosa Canyon Bridge, located in New Mexico, has been used by Straser and Kiremidjian [7] and Lynch et al [8] to validate their respective wireless sensor prototypes. Chung et al [9] have installed a network of wireless sensors upon a footbridge on the University of California-Irvine campus to monitor bridge responses to footfall excitation. Ou et al [10] report on their use of eight Crossbow MICA Motes, a common commercial wireless sensor, to measure the wind-induced response of the Di Wang Tower, Guangdong, China. Field testing will continue to be an important validation environment for assessing the true merits and limitations of wireless monitoring systems as the technology matures.

In this study, a wireless sensor for monitoring large-scale civil structures is designed from state-of-the-art embedded system components. The wireless sensor is intended to be an autonomous data acquisition node within a wireless structural monitoring system. In addition to a high-resolution ADC and ample on-board memory, a microcontroller core is provided to allow the wireless sensor to process its own measurement data. To showcase the performance of the wireless monitoring system, the Geumdang Bridge located in Icheon, Korea, was instrumented with a dense network of wireless sensor prototypes. During forced vibration testing of the Geumdang Bridge, three testing goals were established: (1) compare the time-history acceleration response of the wireless monitoring system to that collected from a commercial tethered monitoring system, (2) illustrate the embedded computing capabilities of the wireless sensors, and (3) validate the accuracy of synchronizing the wireless network using beacon signals. To improve the performance of the individual wireless sensors, a signal conditioning circuit was designed to amplify and band-pass accelerometer outputs. In addition to using the high-resolution wireless monitoring system to record bridge accelerations, the computational resources of the individual wireless sensors are leveraged for local data processing. The distributed computing resources offered by the wireless monitoring system will be used to estimate the modal parameters of the bridge including modal frequencies and mode shapes.

\section{Wireless sensors and signal conditioning}

\subsection{Hardware design of a wireless sensor}

A wireless sensor is simply an integration of a radio with a traditional sensing transducer (e.g. strain gage, displacement sensor, among others). Many different wireless communication technologies can be considered for this application, but the majority of today's radios are digital. In other words, data must first be encoded in a digital format prior to its modulation on the wireless channel. Therefore, by virtue of being wireless, the sensor requires two other important elements in its design. First, an ADC is needed to convert analog sensor outputs to a digital form. Second, a microcontroller is needed to collect digital sensor data from the ADC and modulate the data on the wireless channel. It is this integration of an ADC and microcontroller with the sensor that distinctly sets wireless monitoring systems apart from traditional tethered systems. For example, the collocation of computational power with sensors offers the unique opportunity to process data locally as opposed to processing data at a centralized data repository. Unfortunately, a challenge associated with distributed analog-to-digital conversion is that wireless sensors are no longer tied to the data repository clock; this renders time synchronization more difficult to attain in a wireless monitoring system.

The wireless sensor prototype proposed for use in this study was designed using the three major functional elements that define its functionality: wireless communication channel, analog-to-digital converter and embedded computing power. To ensure wireless sensors can be spaced an adequate distance apart in large civil structures, long-range wireless radios are needed. The Maxstream 9XCite wireless radio was selected for the wireless prototype for two reasons. First, this radio operates on the $900 \mathrm{MHz}$ unlicensed industrial, scientific, and medical (ISM) radio band. Second, the radio strikes a good balance between communication range ( $300 \mathrm{~m}$ maximum line-of-sight range) and power consumption (275, 175 and $0.1 \mathrm{~mW}$ when transmitting, receiving, and powered-down, respectively) [11]. Spread spectrum modulation ensures 


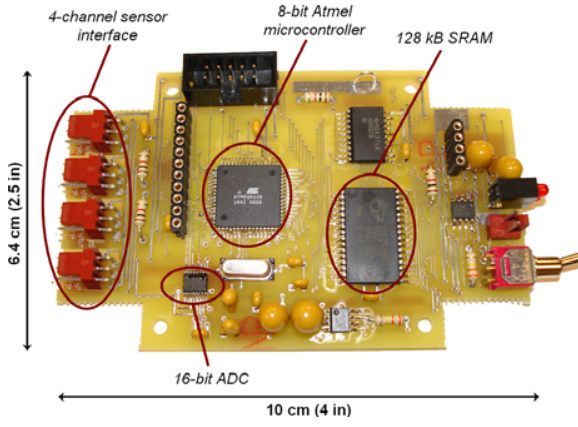

(a)

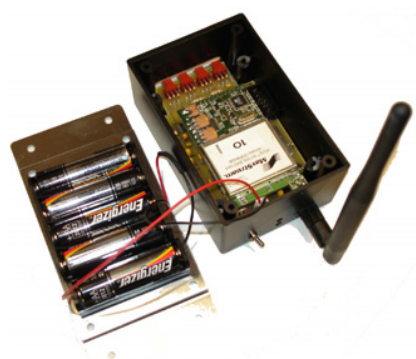

(b)

Figure 1. Wireless sensor prototype for structural monitoring applications: (a) printed circuit board and (b) fully assembled wireless sensor with the 5 AA battery pack revealed.
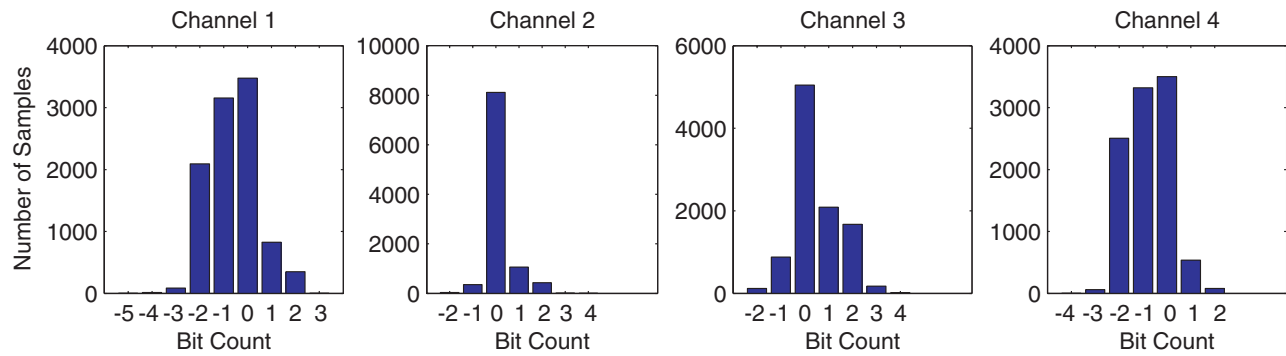

Figure 2. Histogram of integer difference in the wireless sensor ADC when measuring a battery output (with a nominal voltage of 2 V).

that the radio is also robust to interference. To digitize data prior to communication, a four-channel 16-bit ADC was selected for integration in the wireless sensor design. The Texas Instruments ADS8341 ADC can simultaneously sample four independent sensors whose outputs range from 0 to $5 \mathrm{~V}$. The maximum sample rate of the ADC is $100 \mathrm{kHz}$, which is well above most structural monitoring requirements. The last element of the wireless sensor design is its computational core. The core is designed around a low-power 8-bit microcontroller. The 8-bit Atmel AVR ATmega128 microcontroller was selected for its numerous onchip peripherals including $128 \mathrm{kB}$ of flash read-only memory (ROM). However, with only $4 \mathrm{kB}$ of on-chip random access memory (RAM), an additional $128 \mathrm{kB}$ of external static RAM (SRAM) is included in the computational core design for storage of sensor data.

To package the selected hardware components into a compact wireless sensor prototype, a two-layer printed circuit board was designed and fabricated. As shown in figure 1(a), all electrical components are surface mounted to the printed circuit board. Since the 9XCite wireless radio consists of its own circuit board, the radio is stacked above the two-layer printed circuit board that houses the other hardware elements. These stacked printed circuit boards are then combined with a portable power source (e.g. batteries) and fastened to the interior of a hardened plastic container that offers protection from the harsh field environment. The final, fully assembled wireless sensor is roughly $6.4 \times 10 \times 8 \mathrm{~cm}^{3}$, as shown in figure 1(b). With power harvesting technologies still in their infancy, batteries represent the likely power source of the wireless sensors. The current prototype employs five
AA lithium-ion batteries to provide a $5 \mathrm{~V}$ referenced voltage supply. A current meter is used to measure the electrical current drawn from the battery source; with an average current of $77 \mathrm{~mA}$, the wireless sensor power is $385 \mathrm{~mW}$.

\subsection{Signal conditioning for low-amplitude structural responses}

A wireless monitoring system must be capable of recording both ambient and forced (e.g. seismic) structural vibrations. With ambient vibrations typically defined by small amplitudes, high-resolution ADCs are normally employed in structural monitoring systems. For example, commercial monitoring systems offer 16-bit or higher converter resolutions. To provide similar performance, the wireless sensor prototype proposed in this study includes a 16-bit ADC. However, placement of an ADC upon a two-layer circuit leaves the ADC vulnerable to electrical noise present in the circuit. In particular, the opening of transistor gates in the digital circuit elements (e.g. microcontroller) subjects analog circuit elements, such as the ADC, to noise [12]; the end result is a reduction in the ADC resolution.

To determine the effective ADC resolution, a stable noisefree voltage source is interfaced to the wireless sensor. In this study, an AA battery with a nominal voltage of $1.5 \mathrm{~V}$ is employed. The wireless sensor samples the ADC to collect a 10000-point time-history record of the battery voltage. In theory, the recorded time-history should consist of a constant value with only the least significant bit of the digital data being unstable (toggling between 0 and 1). However, if more significant bits are also toggling, this is evidence of circuit 
noise interfering with the function of the ADC. The integer span of this noise can be used to assess the effective resolution of the ADC. As shown in figure 2, histograms of the range of integer values obtained by the ADC when sampling the AA battery are presented for each sensor channel. For example, noise in the first ADC sensor channel spans eight integer values. This implies that the three least significant bits of the $\mathrm{ADC}$ are unstable. In this case, the effective ADC resolution would be 13 bits. Similar results are found on the three other sensor channels.

The reduced ADC resolution of the wireless sensor will be most pronounced when sampling low-amplitude vibration data. Consider the case of an accelerometer, whose sensitivity is $0.7 \mathrm{~V} / \mathrm{g}$ and noise floor is $0.15 \mathrm{mg}$, which is interfaced to the wireless sensor. The lowest measurable voltage of the 13-bit $\mathrm{ADC}$, due to quantization error in the last three bits, is roughly $6.1 \times 10^{-4} \mathrm{~V}\left(=5 \mathrm{~V} / 2^{13}\right)$. However, the voltage noise floor of the accelerometer is $1.05 \times 10^{-4} \mathrm{~V}(=0.7 \mathrm{~V} / g \times 0.15 \mathrm{mg})$ which is well below the quantization error of the 13-bit ADC. Clearly, the quantization error of the ADC controls the quality of the data recorded from the accelerometer. To gain the benefit of the low-noise accelerometer, amplification can be employed. For example, if the accelerometer output is amplified by a factor of 20, the effective sensitivity of the accelerometer is $14 \mathrm{~V} / \mathrm{g}$. As a result of the larger sensitivity, the voltage noise floor of the accelerometer is now $21 \times 10^{-4} \mathrm{~V}$ which is three times greater than the quantization error of the ADC. By employing amplification, the noise floor of the accelerometer would then control the data quality. It should be checked that the sensor output does not exceed the ADC input range (0-5 V for the wireless sensor) when amplified.

To render the wireless sensors suitable for use in ambient structural vibration studies, a signal conditioner is proposed to condition the voltage output of accelerometers prior to connection to the wireless sensor. As shown in figure 3, a small $\left(3.5 \times 5 \mathrm{~cm}^{2}\right)$ signal conditioning circuit is designed. The signal conditioner is designed to perform three tasks: (1) amplify, (2) band-pass, and (3) mean-shift sensor outputs. Three different amplification factors, selected using a three-pin dip switch, are included in the signal conditioner design $(\times 5, \times 10$, and $\times 20)$. After amplification, the sensor outputs are band-pass filtered using a four-pole Bessel filter with a pass band spanning from 0.014 to $25 \mathrm{~Hz}$. A Bessel filter is selected to avoid phase-shift problems typical of Butterworth band-pass filters [12]. The last element of the signal conditioning circuit is the shifting of the sensor output to a $2.5 \mathrm{~V}$ mean. This last feature is particularly useful for sensors whose outputs span from negative to positive voltages (e.g. -5 to 5 V). Previously, such sensors could not be used because their outputs partially fall outside the range of the ADC (0-5 V); now, the original $0 \mathrm{~V}$ mean would be shifted to $2.5 \mathrm{~V}$. An electrical schematic of the signal conditioning circuit is presented in figure 4 .

\subsection{Embedded firmware}

Software embedded in the microcontroller core of the wireless sensor is necessary to automate its behavior when installed in a structure. Termed firmware, this software is intended to perform data acquisition tasks using the wireless sensor hardware. In addition to data acquisition, firmware is

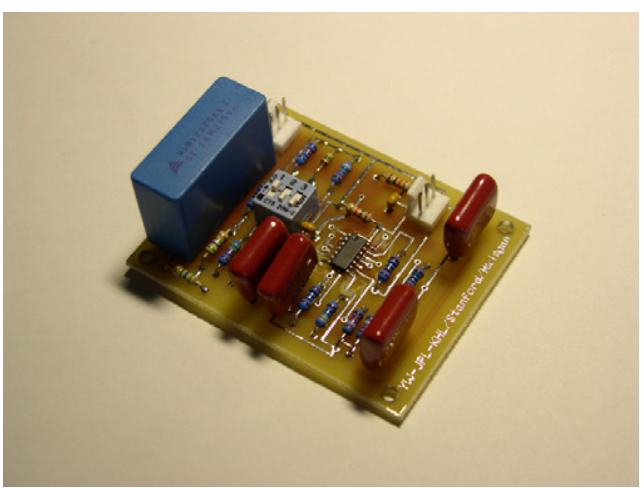

Figure 3. Signal conditioning circuit to amplify and band-pass filter sensor outputs.

also written to process measurement data at the sensor. The embedded firmware is structured using a multi-layer approach. At the lowest layer is the wireless sensor's real-time operating system (RTOS) which will directly operate hardware (e.g. operate the ADC at a precise sample rate). Software that manages and processes sensor data resides on a second upper layer of the firmware architecture. The second tier of firmware can interact with the first tier through functions published by the RTOS. In this study, the embedded firmware is written in $\mathrm{C}$, which is a common high-level programming language widely used in the embedded system field. The features of both layers will be briefly highlighted herein, but additional details are provided by Wang et al [13] and Lynch et al [14].

2.3.1. Multi-threaded real-time operating system. The operating system operates the wireless sensor hardware in order to perform tasks such as operate the ADC, manage data in memory, and establish reliable communications with other sensors. The operating system is written in a modular fashion with distinct software modules dedicated to specific hardware services. For example, one software module operates the wireless radio while another module is used to read sensor data from the ADC. The operating system is designed to be real-time; in other words, it can guarantee that certain tasks are performed on a precise schedule without delay [15]. The ability to perform tasks, such as data sampling, in real-time is a requirement for any data acquisition system. If the sample rate is not held fixed (not real-time), subsequent data processing by the wireless sensor could provide inaccurate results. The operating system takes advantage of the hardware interrupt services included in the ATmega128 microcontroller to guarantee that real-time tasks are performed upon being called. Interrupts will preempt any non-real-time task to service the software routine associated with the interrupt. An additional feature of using interrupts is that the operating system can multi-task; in other words, multiple code routines can execute simultaneously.

The wireless sensor firmware allows the wireless sensor to collect structural response data in one of two modes of operation: ring-buffered data streaming and local data storage. The first mode of operation structures the internal RAM memory of the ATmega128 as four ring-buffers (one for each sensor channel) in which data are stored as the ADC is serviced in real-time. Each wireless sensor is provided with a window 


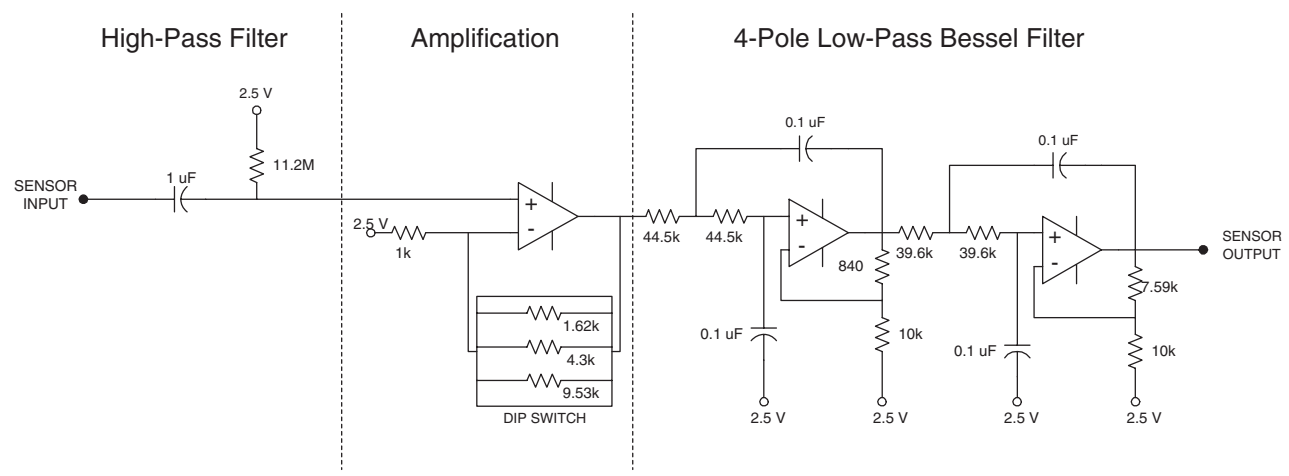

Figure 4. Electrical circuit schematic of the signal conditioner.

of time in which the wireless bandwidth is available for use. When that window occurs, the wireless sensor will transmit data from each ring buffer to the remainder of the wireless sensor network. As the wireless sensor is transmitting its buffered data, it continues to collect data from the ADC and stores them in the ring buffer. When the time window closes, the wireless sensor ceases its communication and cedes the channel to another wireless sensor. This medium access control scheme makes efficient use of the limited wireless bandwidth and provides each wireless sensor equal access to the channel. However, as the number of nodes in the wireless monitoring system increases, the sample rate of the wireless monitoring system should be reduced to ensure the ring buffers do not overwrite prior to the arrival of the communication window. The second mode collects structural response data for a prescribed period (the on-board SRAM memory can store up to 64000 data points at one time) at a sample rate specified by the wireless monitoring system end user. After the wireless monitoring system completes its collection of data, each sensor is given a window of time in which it can wirelessly transmit the entirety of its stored data.

A reliable wireless communication protocol that ensures no data are lost has been included in the design of the embedded firmware. The communication protocol empowers the wireless monitoring system coordinator (e.g. a laptop computer serving as the system data repository) to control communication between wireless sensors and itself. During both modes of operation, the wireless sensors in the network must synchronize themselves prior to the collection of response data. To initiate the collection of structural response, the wireless monitoring system coordinator issues a wireless beacon signal. The wireless sensors are designed to begin collecting data, without delay, upon receipt of the beacon signal. After the wireless sensors begin to collect data, they each communicate an acknowledgment to the central coordinator. If the central coordinator does not receive acknowledgments from all of the wireless sensors, the procedure is repeated and another beacon signal initiated. This method of time synchronization is similar to synchronization methods employed in other wireless communication protocols [16]. After the coordinator confirms that all of the wireless sensors are synchronized and have begun to collect structural response data, the coordinator can then issue command signals to individual wireless sensors to send their data. Upon receipt of that command, the wireless sensor responds with an acknowledgment that it has received the command followed by a wireless packet containing the data requested (e.g. raw sensor or post-processed data). If the central repository does not receive either the acknowledgment or data packet, the repository will reissue the same command to the wireless sensor. The communication protocol proposed has been thoroughly tested in the field and has proven highly reliable with no data lost during operation of the wireless monitoring system.

2.3.2. Local data-processing software. The use of wireless sensors to self-interrogate measurement data is what has led to their label as 'smart' sensors [6]. Many advantages are associated with local data processing including the reduction of data glut through parallel processing of data. But perhaps of greater motivation is that local data processing is significantly more power-efficient than wirelessly transmitting long records of time-history data to data repositories [17]. A number of engineering analysis algorithms have been previously embedded in the cores of wireless sensors, including autoregressive (AR) model fitting, wavelet transforms, among others [14]. In this study, two data processing algorithms will be locally executed by the wireless sensors. First, the CooleyTukey implementation of the fast Fourier transform (FFT) is executed by the wireless sensors to convert time-history response data to the frequency domain where structural modal properties can be identified. The complex-valued Fourier spectra can be wirelessly communicated or locally stored in memory for further data processing. The second embedded algorithm is a peak-picking scheme that can identify modal frequencies from peaks in the Fourier spectra.

In addition to determination of modal frequencies, the mode shapes of the structure are also of interest. In many monitoring studies, precise knowledge of the structure loading does not exist. However, traffic loads on bridges are often assumed to be sufficiently random that the input excitation is considered white noise [18]. Based on this assumption, the Fourier spectra calculated from the bridge response data at sensor location $k(k \in[1, n]$ since it is assumed that there are, in total, $n$ sensors installed) is considered to be the frequency response function (FRF), $H_{k}(\mathrm{j} \omega)$, of the structure. If the structure is lightly damped $(\zeta<10 \%)$ and the modes are well separated, the mode shapes can be determined based upon the imaginary components of the different FRFs. The imaginary 
component of the FRFs at modal frequency, $\omega_{i}$, and at sensor locations 1 through $n$, can be assembled as

$$
\left\{\phi_{i}\right\}=\left\{\begin{array}{c}
\operatorname{imag}\left[H_{1}\left(\mathrm{j} \omega_{i}\right)\right] \\
\operatorname{imag}\left[H_{2}\left(\mathrm{j} \omega_{i}\right)\right] \\
\vdots \\
\operatorname{imag}\left[H_{n}\left(\mathrm{j} \omega_{i}\right)\right]
\end{array}\right\}
$$

to yield the $i$ th mode shape, $\phi_{i}$ [19]. When the input to the structure is unknown, the vector of imaginary components is termed the operational deflection shape (ODS) of the system. The peak picking algorithm in the wireless sensors identifies the modal frequencies of the structure. Once these frequencies are estimated, the wireless sensors transmit the imaginary components at those frequencies so that the wireless sensor network can assemble the mode shapes. This approach to mode shape estimation is inherently decentralized and well suited for the distributed computing paradigm offered by the wireless monitoring system. It is also a power-efficient approach to mode shape estimation because no time-history data are wirelessly transmitted; rather, only estimated mode shape values at each sensor location are transmitted.

\section{Experimental bridge structure}

\subsection{Geumdang Bridge}

The Geumdang Bridge, located in the vicinity of Icheon, Korea, is selected as a convenient field structure that can be used to validate the proposed wireless monitoring system. The Geumdang Bridge is one of three bridges along a $7.7 \mathrm{~km}$ test road recently constructed parallel to the Jungbu Inland Highway (construction was completed in 2002). Designed and managed by the Korea Highway Corporation (KHC), the two-lane test road employs 1897 sensors to measure the performance of three types of pavement system constructed along the road length (asphalt, plain concrete, and reinforced concrete) [20]. Although the test road pavement is densely instrumented, the three bridges (Geumdang Bridge, Yondae Bridge, and Samseung Bridge) that carry traffic across irrigated agricultural valleys are not continuously monitored. Rather, Lee et al [21] have performed short-term vibration studies of the Geumdang and Samseung bridges to assess their load carrying capacities. A convenient feature of the test road that is important in this study is that the $\mathrm{KHC}$ has the ability to open or close the road to highway traffic. In this study, the $\mathrm{KHC}$ closed the test road and permitted only vehicles intended for testing to enter.

The Geumdang Bridge, with a total length of $273 \mathrm{~m}$, was designed using two different section types. First, the northern-most spans of the bridge carry traffic loads using a deck-girder section design. In total, four independent spans (with span lengths of $31,40,40$, and $40 \mathrm{~m}$, respectively) were designed using a $27 \mathrm{~cm}$ concrete deck supported by four pre-cast concrete girders. The southern portion of the bridge carries highway traffic using a continuous $122 \mathrm{~m}$ posttensioned concrete box girder section. The box girder is supported along its length by three piers in addition to a bridge abutment structure. The placement of the bridge piers provide the box girder section with a main span of $46 \mathrm{~m}$ and two side spans of $38 \mathrm{~m}$. The dimensional details of the Geumdang Bridge box girder section are presented in figure 5 .

\subsection{Instrumentation strategy}

The $122 \mathrm{~m}$ post-tensioned concrete box girder section of the Geumdang Bridge was selected for instrumentation using two monitoring systems. The first system was a traditional tethered monitoring system with piezoelectric accelerometers interfaced to a centralized data acquisition unit using coaxial wires. The second monitoring system was a wireless monitoring system assembled from the proposed wireless sensors with low-cost capacitive accelerometers attached. Both monitoring systems, installed on a temporary basis, were configured to record the vertical acceleration response of the bridge during ambient and forced excitations. In this study, the tethered monitoring system served as a baseline monitoring system to which the performance of the wireless monitoring system could be directly compared. In particular, the performance features investigated include data quality and time synchronization.

The Geumdang Bridge was instrumented on two separate occasions. First, the tethered and wireless monitoring systems were installed within the accessible interior spaces of the box girder during December 2004 (December 5-7, 2004). During the December tests, the wireless monitoring system consisted only of capacitive accelerometers interfaced to the wireless sensor prototypes. As a result of a higher than desired noise floor inherent in the wireless sensors, the bridge was again instrumented in July 2005 (July 25-27, 2005). To improve the performance of the wireless monitoring system, signal conditioning circuitry was included with each wireless sensor to amplify the output of the accelerometers. The location of the wireless and tethered monitoring systems' accelerometers for both sets of tests (December and July) are presented in figure 6 . During the December 2004 tests, a total of 14 wireless sensors was installed parallel to the 16 accelerometers interfaced to the tethered monitoring system. For the tests performed in July 2005, a new sensor configuration was adopted for the 14 wireless sensors. For the tethered monitoring system installed in July 2005, only 13 accelerometers were installed in the bridge.

The tethered monitoring system employed PCB Piezotronics 393B12 integrated circuit piezoelectric (ICP) accelerometers to record the vertical acceleration response of the bridge. This seismic accelerometer has a sensitivity of $10 \mathrm{~V} / g$ and range of $0.5 \mathrm{~g}$ peak-to-peak. The 393B12 accelerometer is well suited for use in ambient vibration applications because of its low root mean square noise floor $(8 \mu g)$ [22]. To provide a constant current excitation to the ICP accelerometers, a 16-channel PCB Piezotronics 481A03 signal conditioner was employed. The 481A03 signal conditioner can simultaneously amplify (programmable up to a gain of 200) and filter (programmable 8th-order elliptical low pass) accelerometer outputs on 16 channels. The conditioned piezoelectric accelerometer outputs were then digitally recorded by a laptop using the National Instruments 6062E 12-bit data acquisition card. As shown in figure 6 , the data acquisition equipment of the tethered monitoring system was located at the southern entrance to the box girder.

The wireless monitoring system installed in the Geumdang Bridge was assembled from the wireless sensor prototypes. To record the vertical acceleration response of the bridge during forced vibration testing, one accelerometer was 
Performance monitoring of the Geumdang Bridge using a dense network of high-resolution wireless sensors

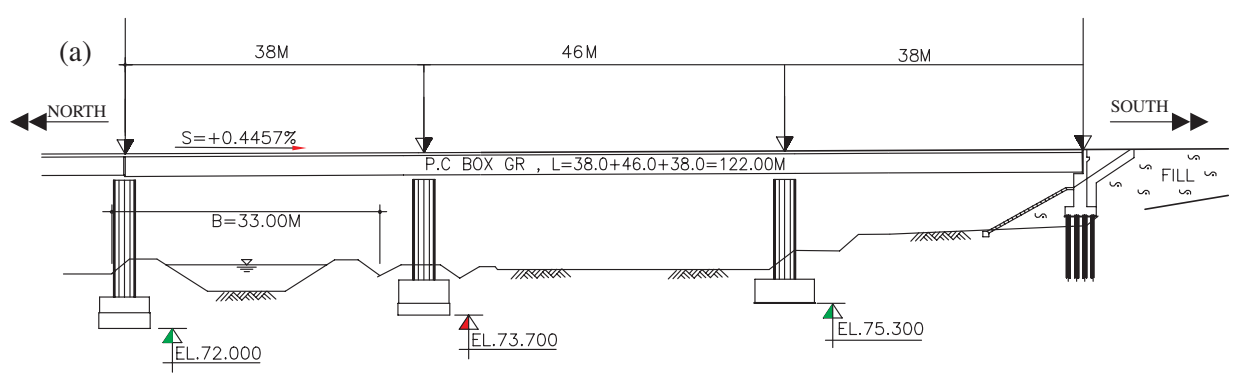

(b)

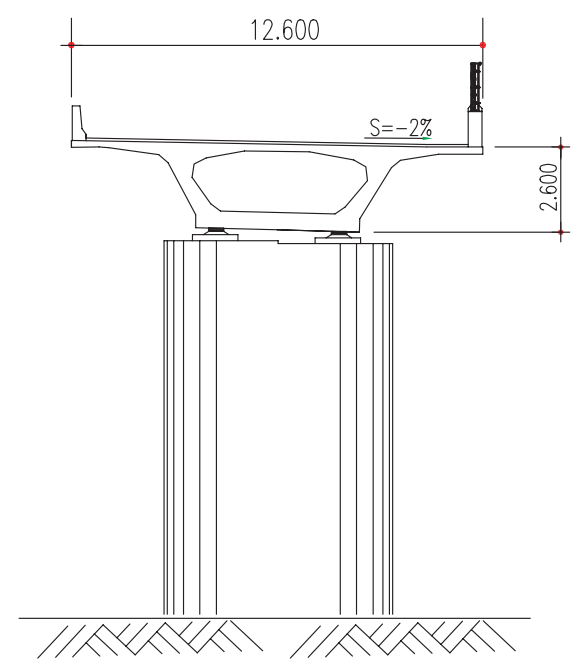

(c)

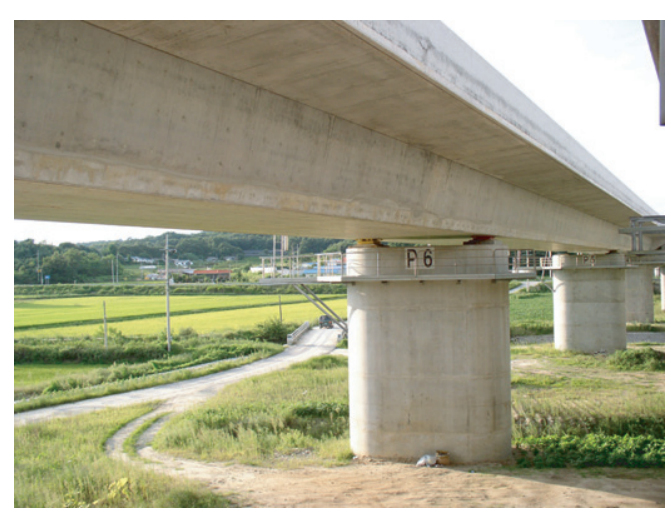

Figure 5. Post-tension concrete box girder span of the Geumdang Bridge: (a) elevation view, (b) typical cross-section geometry, and (c) perspective view.

(a)
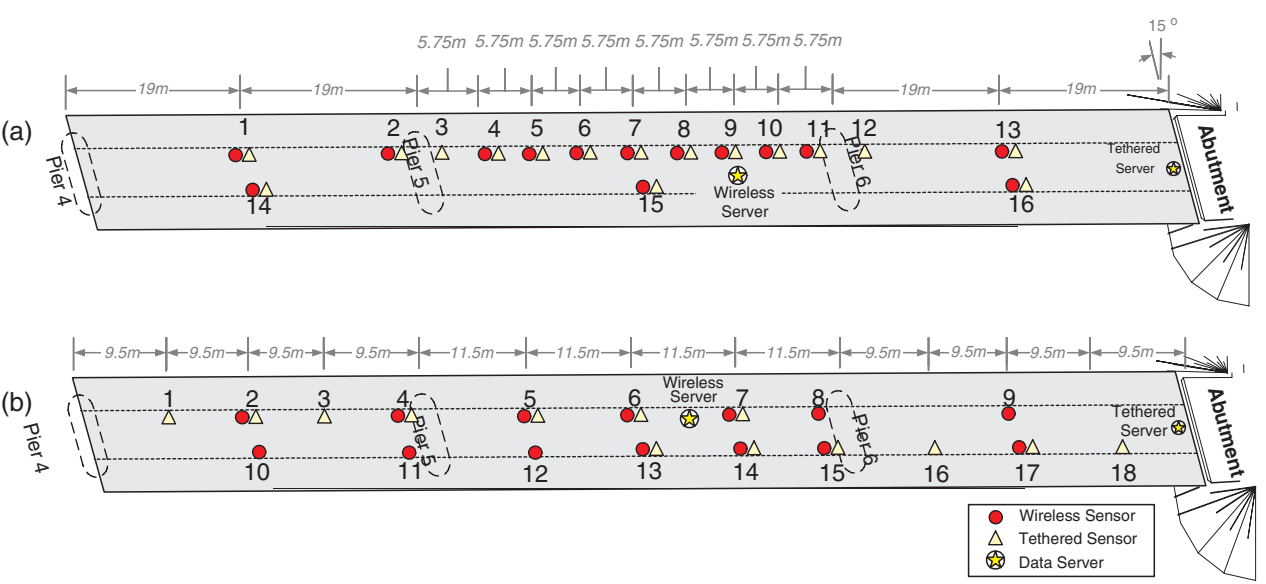

Figure 6. Location of wireless and tethered accelerometers inside the Geumdang Bridge box girder: (a) December 2004 and (b) July 2005.

attached to each wireless sensor. The low-cost PCB Piezotronics 3801D1FB3G MEMS capacitive accelerometers were selected for use with the wireless monitoring system. The sensitivity of the accelerometer is $0.7 \mathrm{~V} / \mathrm{g}$ and its dynamic range is $3 g$ peak-to-peak. The PCB 3801 accelerometer is not as accurate as the PCB 393 accelerometer at low accelerations because of a relatively high noise floor (150 $\mu \mathrm{g})$ [23]. As shown in figure 6 , a laptop computer to command the wireless sen- sor network and to receive response data was placed near the center of the box girder during both sets of vibration tests.

\section{Forced vibration testing}

During both sets of load tests (December 2004 and July 2005), the bridge was intentionally loaded using a series of trucks driven over the bridge at constant velocities. In particular, 
Vertical Acceleration - Tethered Sensor \#8
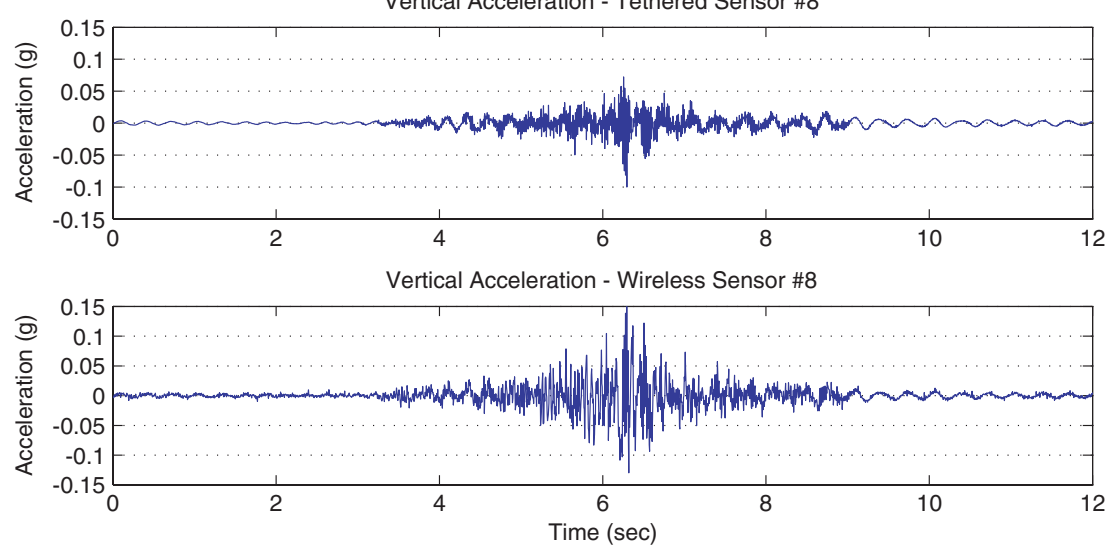

Figure 7. Acceleration time-history response of the Geumdang Bridge at sensor location \#8 corresponding to a 40 ton truck crossing at $80 \mathrm{~km} \mathrm{~h}^{-1}$ (December 2004).

three trucks were employed with three different weights (15, 30 , and 40 tons) precisely measured prior to their arrival at the bridge site. As previously discussed, access to the test road was restricted by the KHC so that only the calibrated trucks were on the road during testing. The trucks were used to dynamically excite the instrumented box girder by driving the trucks, one at a time, over the bridge at a fixed velocity in the southward direction. Three velocities were used during testing: 40, 60, and $80 \mathrm{~km} \mathrm{~h}^{-1}$. After the trucks traversed the bridge, they were driven over the bridge at $10 \mathrm{~km} \mathrm{~h}^{-1}$ to return to their original position on the northern side of the bridge.

While the trucks were driven over the bridge, the tethered and wireless monitoring systems were simultaneously used to record the vertical acceleration response of the Geumdang Bridge box girder. The tethered monitoring system is capable of sampling at any sample rate desired, but in this study, the system was configured to sample acceleration response data at $200 \mathrm{~Hz}$. During forced vibration testing, a gain of 10 was selected for the 481A03 signal conditioner. The sample rate adopted by the wireless monitoring system depends upon its mode of operation. When operating in ring-buffered data streaming mode, the maximum sample rate prescribed for each of the 14 wireless sensors is $70 \mathrm{~Hz}$ [13]. However, if the wireless sensors are operated in the local data storage mode, they are commanded to sample data at $200 \mathrm{~Hz}$.

\subsection{Measured acceleration without signal conditioning}

The objective of the vibration tests performed during December 2004 was to compare the quality of the acceleration response data recorded by the wireless monitoring system to the response data obtained by the tethered system. The wireless monitoring system records the bridge response without external signal conditioning circuitry. During the tests, the calibrated trucks were driven one at a time over the Geumdang Bridge with the bridge response measured by both monitoring systems. During multiple days of testing, the communication protocol of the wireless monitoring system proved successful in obtaining bridge response data with no data losses reported. Figure 7 presents the time-history acceleration response recorded while a 40 ton truck crosses the

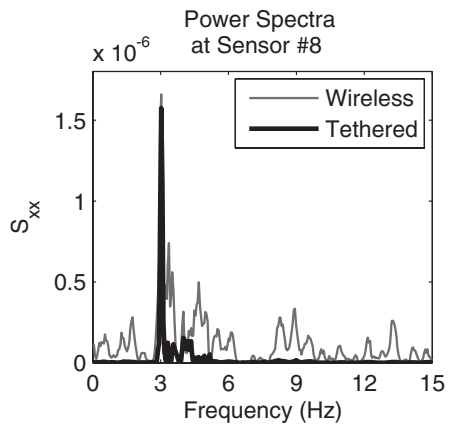

Figure 8. Power spectra function of the acceleration response at sensor location \#8 (December 2004).

bridge at $80 \mathrm{~km} \mathrm{~h}^{-1}$. As seen, strong similarities exist in the time-history records. However, some significant discrepancies are found between the peak accelerations recorded, especially in the time frame when the truck is over the center span of the instrumented box girder bridge (between 5 and $7 \mathrm{~s}$ ). Specifically, the peak acceleration recorded at $t=6.295 \mathrm{~s}$ is $0.08 \mathrm{~g}$, versus $0.15 \mathrm{~g}$ as measured by the tethered and wireless monitoring systems, respectively. As expected, the wireless monitoring system records have elevated acceleration amplitudes as a result of the system noise floor being higher than that of the tethered system. To better understand the noise properties of the wireless monitoring system, the power spectra of both time histories were calculated, as shown in figure 8 . The primary modal frequency of the instrumented span is evident at $3 \mathrm{~Hz}$, but the power spectra corresponding to the wireless monitoring system show the spectra elevated at non-modal frequencies as a result of noise.

To better quantify the noise inherent in the wireless monitoring system, the free-vibration response of the Geumdang bridge was analyzed. The free-vibration response of the box girder begins after the truck is completely removed from the bridge, which is approximately $9 \mathrm{~s}$ after the bridge begins to experience vibrations due to the truck. With the span's first mode having a high participation factor, the freevibration response exhibits a sinusoidal behavior with a period of $0.33 \mathrm{~s}$ and an amplitude envelope slowly decaying due 

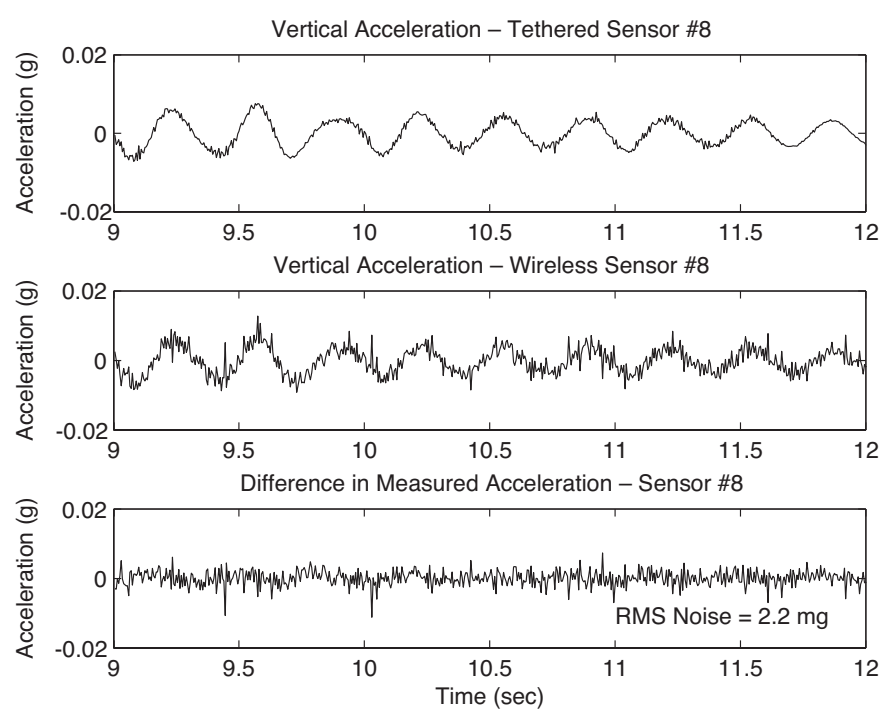

Figure 9. Free-vibration response recorded by the tethered (top) and wireless (middle) monitoring systems and the difference between the two measured histories (bottom) (December 2004).

to structural damping. Figure 9 presents a 3 s portion of the free-vibration response after $9 \mathrm{~s}$. Noise present in the wireless sensor is clearly evident when comparing the freevibration response time-histories recorded by the two systems. The difference in the measured free-vibration responses is due to the noise inherent in the wireless monitoring system. The root mean square of the difference is $2.2 \mathrm{mg}$, which is well above the noise floor of the wireless sensors' PCB3801 accelerometers $(0.15 \mathrm{mg})$. This result suggests that the likely noise source is quantization error in the analog-to-digital conversion performed by the wireless sensor's ADC. The noise floor is determined at each sensor location with similar results found. These results suggest that the performance of the wireless monitoring system can be greatly improved by amplification of the PCB3801 accelerometer output; amplification would raise the accelerometer noise floor above that of the ADC quantization error.

\subsection{Integration of signal conditioning with wireless sensors}

Forced vibration testing of the Geumdang Bridge was again performed in July 2005 with the new sensor configuration shown in figure 6(b). To address the limitation of the lower than desired ADC resolution, signal conditioners were now utilized. One signal conditioner was deployed with each wireless sensor to amplify (by a factor of 20) and to band-pass filter $(0.014-25 \mathrm{~Hz})$ the output of the PCB3801 accelerometers. This amplification factor will allow the noise floor of the PCB3801 accelerometers to control the performance of the wireless monitoring system. Provided the peak response of the bridge should be no greater than $200 \mathrm{mg}$, the amplified accelerometer output should remain in the $5 \mathrm{~V}$ input range of the wireless sensors' ADC. A picture of the accelerometer, signal conditioner, and wireless sensor is shown in figure 10 as deployed within the Geumdang Bridge box girder.

During the July 2005 tests, only the 40 ton truck was utilized for load testing. Again, the truck was driven over the bridge at set speeds to induce structural vibrations into

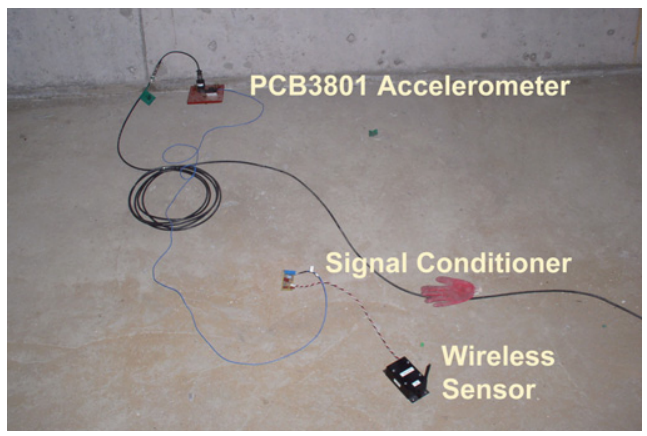

Figure 10. Typical installation of a wireless sensor with a signal conditioner amplifying and filtering the PCB3801 accelerometer output.

the system. In total, five tests were performed; the first three tests drove the truck at $80 \mathrm{~km} \mathrm{~h}^{-1}$ while the fourth and fifth drove the truck at 60 and $40 \mathrm{~km} \mathrm{~h}^{-1}$, respectively. With the signal conditioning circuitry coupled with each wireless sensor, there is a drastic improvement in the quality of the wireless monitoring system response data. The response of the Geumdang Bridge box girder to the 40 ton truck crossing at $40 \mathrm{~km} \mathrm{~h}^{-1}$ (test 5) is presented in figure 11 . Both monitoring systems employed sample rates of $200 \mathrm{~Hz}$ during the collection of the bridge response. Similar to the tests conducted in December 2004, no data loss occurred in the wireless communication channel during the testing of the bridge. Immediately evident from the recorded response is the improved wireless monitoring system resolution. If the response recorded at sensor location \#4 is compared between the two monitoring systems, we see they are nearly identical, as shown in figure 12. Upon closer inspection, if the free-vibration response is again considered, the difference between the response measured by the wireless and tethered monitoring systems can be calculated. The free-vibration response of the Geumdang Bridge is plotted in figure 12 for 

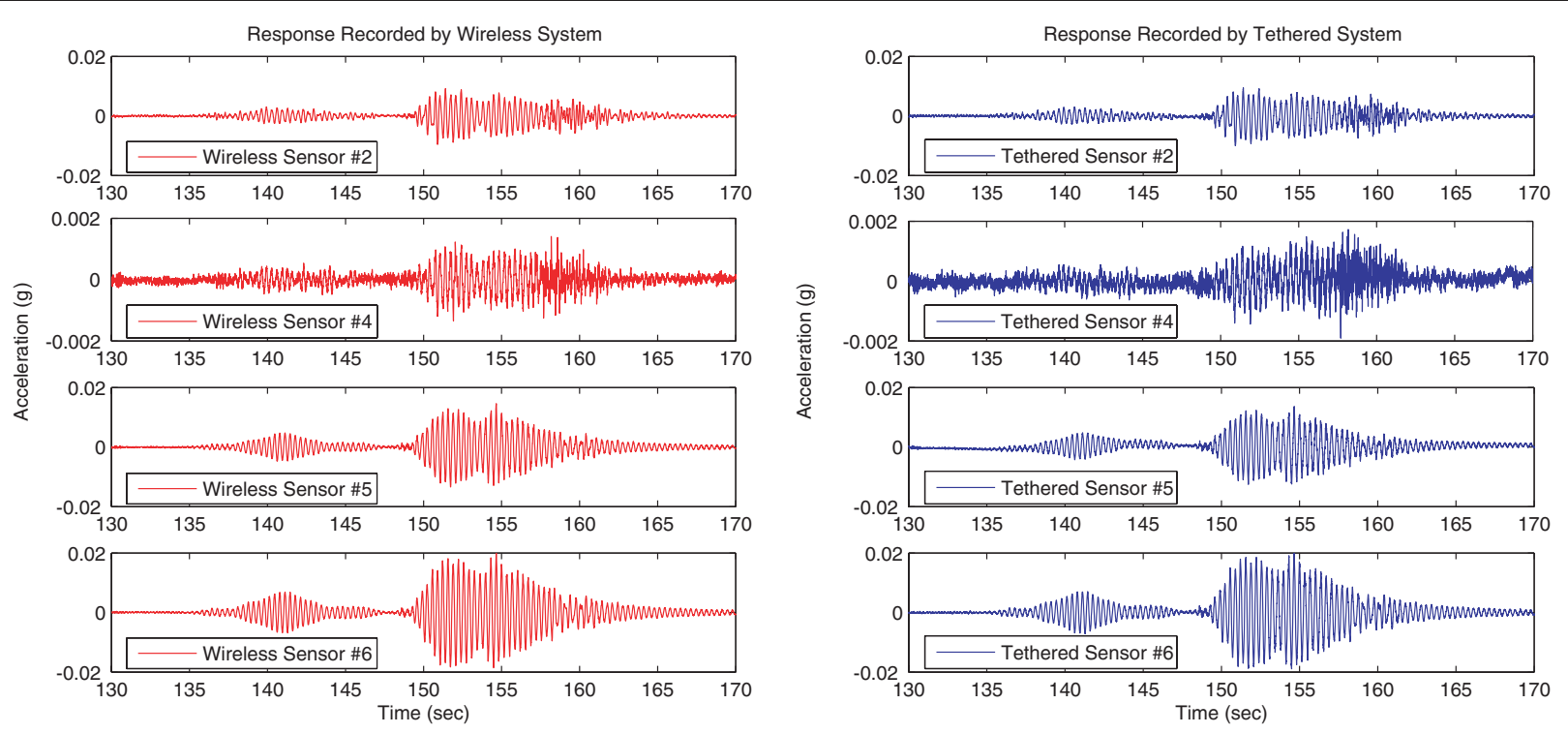

Figure 11. Geumdang Bridge acceleration response to 40 ton truck crossing at $40 \mathrm{~km} \mathrm{~h}^{-1}$ (test 5-July 2005).
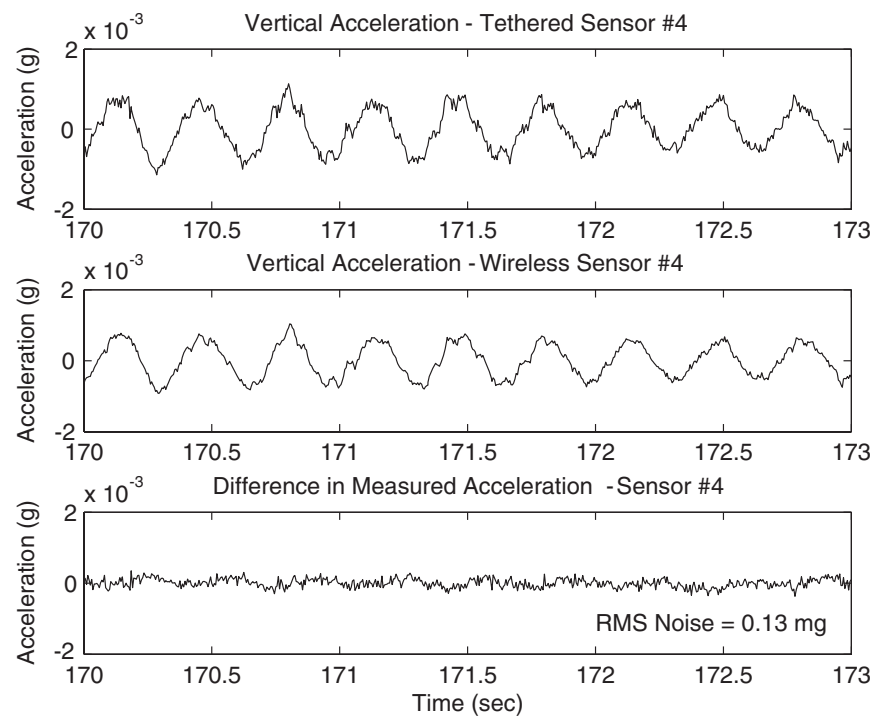

Figure 12. Free-vibration response recorded by the tethered (top) and high-resolution wireless (middle) monitoring systems and the difference between the two measured histories (bottom) (test 5-July 2005).

the response measured from 170 to $173 \mathrm{~s}$. The root mean square of the difference between the wireless and tethered monitoring systems is calculated to be $0.13 \mathrm{mg}$, which is well below that encountered when signal conditioning was not previously employed by the wireless monitoring system (previously measured to be $2.2 \mathrm{mg}$ ). Identical results were obtained at the other sensor locations where both the wireless and tethered monitoring systems had accelerometers. It can be concluded that amplification and filtering of the PCB3801 accelerometer outputs provided the wireless monitoring system with a precision identical to that offered by a commercial structural monitoring system. The high-quality response data collected by the wireless monitoring system could be used to perform a complete modal analysis of the instrumented bridge span.

\section{In-network distributed data processing}

An objective of the wireless monitoring system is to estimate the modal properties of the instrumented bridge span. Using acceleration response data collected during truck excitations, the wireless sensors are each commanded to transform the time-history response data to the frequency domain through the use of their embedded FFT algorithm. Specifically, the wireless sensors are programmed to calculate a 4096-point complex-valued Fourier spectrum from response data stored in its internal memory bank during the July 2005 tests. Fourier spectra of the Geumdang Bridge box girder span are presented in figure 13 for four wireless sensor locations (sensor locations 2, 4, 5 and 6) during the 40 ton truck crossing the bridge at $40 \mathrm{~km} \mathrm{~h}^{-1}$. For comparison, Fourier spectra at the same sensor locations are calculated using the time-history response data 

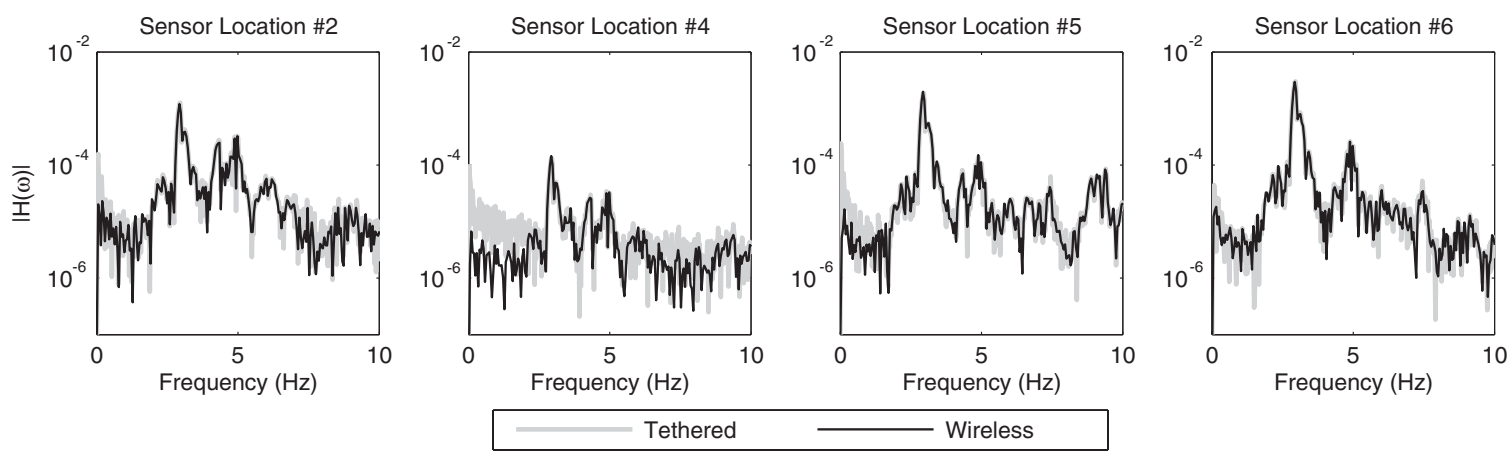

Figure 13. Fourier spectra of the Geumdang Bridge response at four selected sensor locations (test 5-July 2005).
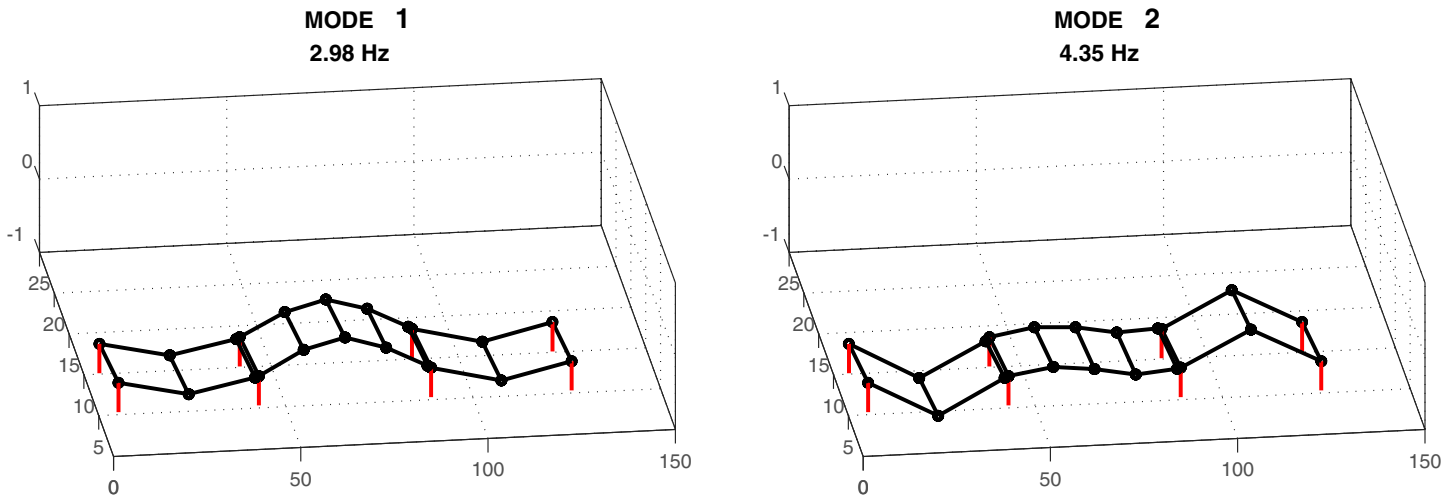

MODE 3

$5.03 \mathrm{~Hz}$

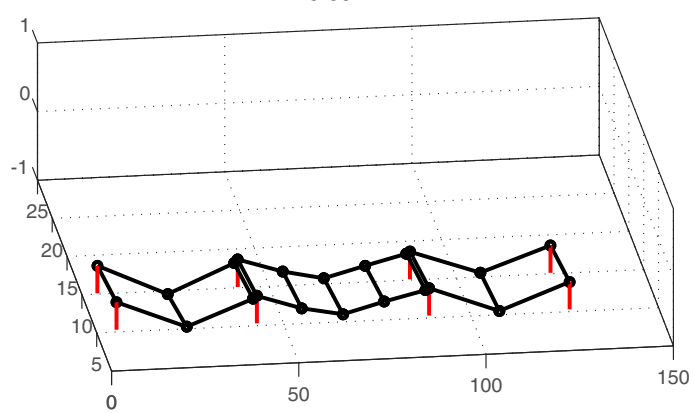

MODE 4

$7.03 \mathrm{~Hz}$

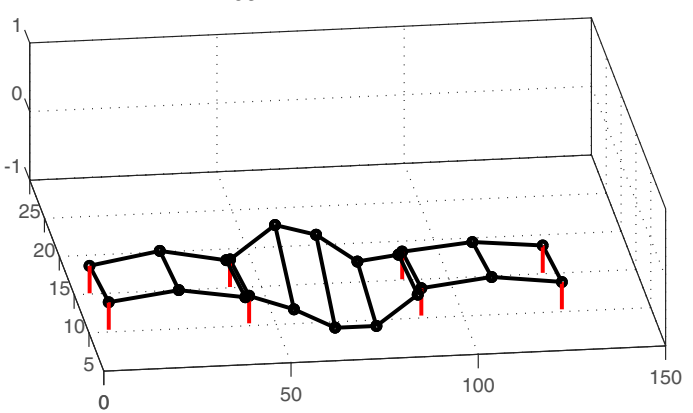

Figure 14. Operational deflection shapes (ODS) calculated by the wireless sensor network during forced vibration loading (test 4 - July 2005).

collected by the tethered monitoring system. As shown in figure 13, the Fourier spectra corresponding to each monitoring system are nearly identical with only minor discrepancies at the lower spectra frequencies $(<1 \mathrm{~Hz})$. Evident from the Fourier spectra are the modal frequencies of the bridge. The embedded peak picking algorithm is adopted by the wireless sensors to determine the frequencies corresponding to spectrum peaks. As summarized in table 1 , the wireless monitoring system is capable of determining the first three modal frequencies for each test $(3.0,4.3$, and $5.0 \mathrm{~Hz})$. Only during test 4 (40 ton truck crossing the bridge at $60 \mathrm{~km} \mathrm{~h}^{-1}$ ) is a fourth modal frequency identified $(7.0 \mathrm{~Hz})$ by the wireless sensors.

Once the modal frequencies are determined by the wireless monitoring system, the imaginary components of the Fourier spectra at the estimated modal frequencies are wirelessly transmitted so that the wireless sensors can determine the operational deflection shapes of the bridge span. Since the properties of the truck loading are not precisely known, the operational deflection shapes are not mode shapes but are only assumed to be dominated by the modes shapes. Figure 14 presents the operational deflection shapes corresponding to the first four modes as calculated by the wireless monitoring system during test 4 . The first three modes are primarily flexural bending modes while the fourth mode is dominated by torsion in the center of the box girder. It should be pointed out that the operational deflection shapes are normalized off-line using MATLAB to have a unit norm.

Using ambient response data (generated from broadband wind and highway traffic on an adjacent bridge span) recorded by the tethered monitoring system in December 
Table 1. Summary of Geumdang Bridge modal frequencies.

\begin{tabular}{|c|c|c|c|c|c|}
\hline \multirow[b]{2}{*}{ Vibration test } & \multirow[b]{2}{*}{ Analysis method } & \multicolumn{4}{|c|}{ Modal frequency $(\mathrm{Hz})$} \\
\hline & & Mode 1 & Mode 2 & Mode 3 & Mode 4 \\
\hline$\overline{\text { Ambient }}$ & $\mathrm{FDD}^{\mathrm{a}}$ & 3.076 & 4.541 & 5.249 & 7.324 \\
\hline Test $1-40$ ton truck @ $80 \mathrm{~km} \mathrm{~h}^{-1}$ & $\mathrm{ODS}^{\mathrm{b}}$ & 3.028 & 4.200 & 4.981 & - \\
\hline Test $2-40$ ton truck @ $80 \mathrm{~km} \mathrm{~h}^{-1}$ & (determined by wireless sensors) & 2.979 & 4.248 & 5.029 & - \\
\hline Test 3-40 ton truck @ $80 \mathrm{~km} \mathrm{~h}^{-1}$ & & 2.979 & 4.297 & 5.029 & - \\
\hline Test $4-40$ ton truck @ $60 \mathrm{~km} \mathrm{~h}^{-1}$ & & 2.979 & 4.346 & 5.029 & 7.032 \\
\hline Test 5-40 ton truck @ $40 \mathrm{~km} \mathrm{~h}^{-1}$ & & 3.028 & 4.249 & 4.932 & - \\
\hline
\end{tabular}

2004, the modes of the bridge are determined using the frequency domain decomposition (FDD) mode shape estimation method [24]. The FDD mode shape estimation method is also widely known as the complex mode indication function (CMIF) method [19]. The FDD estimation method identifies the first four modal frequencies of the instrumented bridge span at 3.1, 4.5, 5.2, and $7.3 \mathrm{~Hz}$, which agree with those determined by the wireless monitoring system in July 2005. Figure 15 presents the first four modes (also normalized to a unit value) determined by the FDD method using MATLAB. Although two different instrumentation schemes were employed during the December and July tests (figure 6), the modes determined from the ambient response data have strong agreement with the operational deflection shapes determined by the wireless monitoring system. Again, the first three modes are seen to be primarily flexural bending modes. However, the fourth mode shape's torsion response is much more dominant than in the fourth operational deflection shape determined by the wireless monitoring system.

To quantify the similarities which exist between the operational deflection shapes and the mode shapes, the modal assurance criterion (MAC) for mode $i$ is calculated using the $i$ th mode shape $\left(\phi_{i, \mathrm{FDD}}\right)$ and the corresponding $i$ th operational deflection shape $\left(\phi_{i, \text { ODS }}\right)[25]$ :

$\mathrm{MAC}_{i}$

$$
=\frac{\left|\sum_{k=1}^{j} \phi_{i, \mathrm{ODS}}(k) \cdot \operatorname{conj}\left(\phi_{i, \mathrm{FDD}}(k)\right)\right|^{2}}{\left(\sum_{k=1}^{j} \phi_{i, \mathrm{ODS}}(k) \cdot \operatorname{conj}\left(\phi_{i, \mathrm{ODS}}(k)\right)\right)\left(\sum_{k=1}^{j} \phi_{i, \mathrm{FDD}}(k) \cdot \operatorname{conj}\left(\phi_{i, \mathrm{FDD}}(k)\right)\right)} .
$$

Since two different sensor configurations are adopted during the two tests, the MAC is determined only at the $j$ locations where both monitoring systems share a common sensor location. Here, $\phi_{i, \mathrm{FDD}}(k)$ designates the mode value at sensor location $k(k \in[1, j])$ of the $i$ th mode as determined by the FDD method. As summarized in table 2, the MAC is used to compare the operational deflection shapes calculated during each of the five vibration tests to the mode shapes determined from the ambient response data collected in December 2004. Strong agreement exists in the first and third modes, with MAC values greater than 0.9 encountered. The second response mode of the bridge is well correlated to the second operational deflection shape, with an average MAC of 0.8. The MAC corresponding to the fourth mode (0.6) confirms the earlier visual observation that the fourth mode shape and fourth operational deflection shape have different types of torsion responses.
Table 2. Modal assurance criteria (MACs) for operational deflection shapes calculated by the wireless sensor network.

\begin{tabular}{lllll}
\hline & \multicolumn{4}{c}{ Modal assurance criteria } \\
\cline { 2 - 5 } Vibration test & Mode 1 & Mode 2 & Mode 3 & Mode 4 \\
\hline Test 1 & 0.975 & 0.765 & 0.950 & - \\
Test 2 & 0.988 & 0.786 & 0.951 & - \\
Test 3 & 0.976 & 0.774 & 0.948 & - \\
Test 4 & 0.989 & 0.813 & 0.954 & 0.6010 \\
Test 5 & 0.983 & 0.846 & 0.890 & - \\
\hline
\end{tabular}

\section{Network time synchronization}

A distinct advantage of tethered monitoring systems is a centralized clock at the data repository which accurately synchronizes time-history data from multiple sensors. Synchronization of response data is necessary to ensure the accuracy of the engineering analyses performed on the response data. In particular, calculation of system modes is sensitive to errors in the synchronization of multi-output structural systems [26]. To address this limitation, the embedded firmware of the wireless monitoring system employs a beacon signal to which independent wireless sensors can synchronize their local clocks. This method, widely used in common wireless communication protocols including IEEE 802.11 and 802.15.4, assumes that the beacon signal delivery time is infinitesimally small [16]. However, delays in the communication channel or delay in the wireless sensor's perception of the beacon can result in minor inaccuracies in this decentralized time synchronization scheme. With a perfectly synchronized tethered monitoring system installed in parallel to the wireless sensors, synchronization errors of the wireless monitoring system can be measured directly.

To quantify the delay in synchronization of a wireless sensor's clock based upon the beacon signal, the time-history response collected by the wireless monitoring system is compared to that of the tethered system. At each sensor location, the wireless time-history response is shifted by various time-step increments $(\Delta t)$, and the vector norm of the difference in the tethered and wireless acceleration response $\left(a_{T}(t)\right.$ and $a_{W X}(t)$, respectively) data is determined:

$$
|e(k)|=\left|a_{T}(t)-a_{W X}(t+k \Delta t)\right| .
$$

The time-step increment, $k$, which minimizes the norm of the error, $|e(k)|$, represents the appropriate alignment of the 
Performance monitoring of the Geumdang Bridge using a dense network of high-resolution wireless sensors
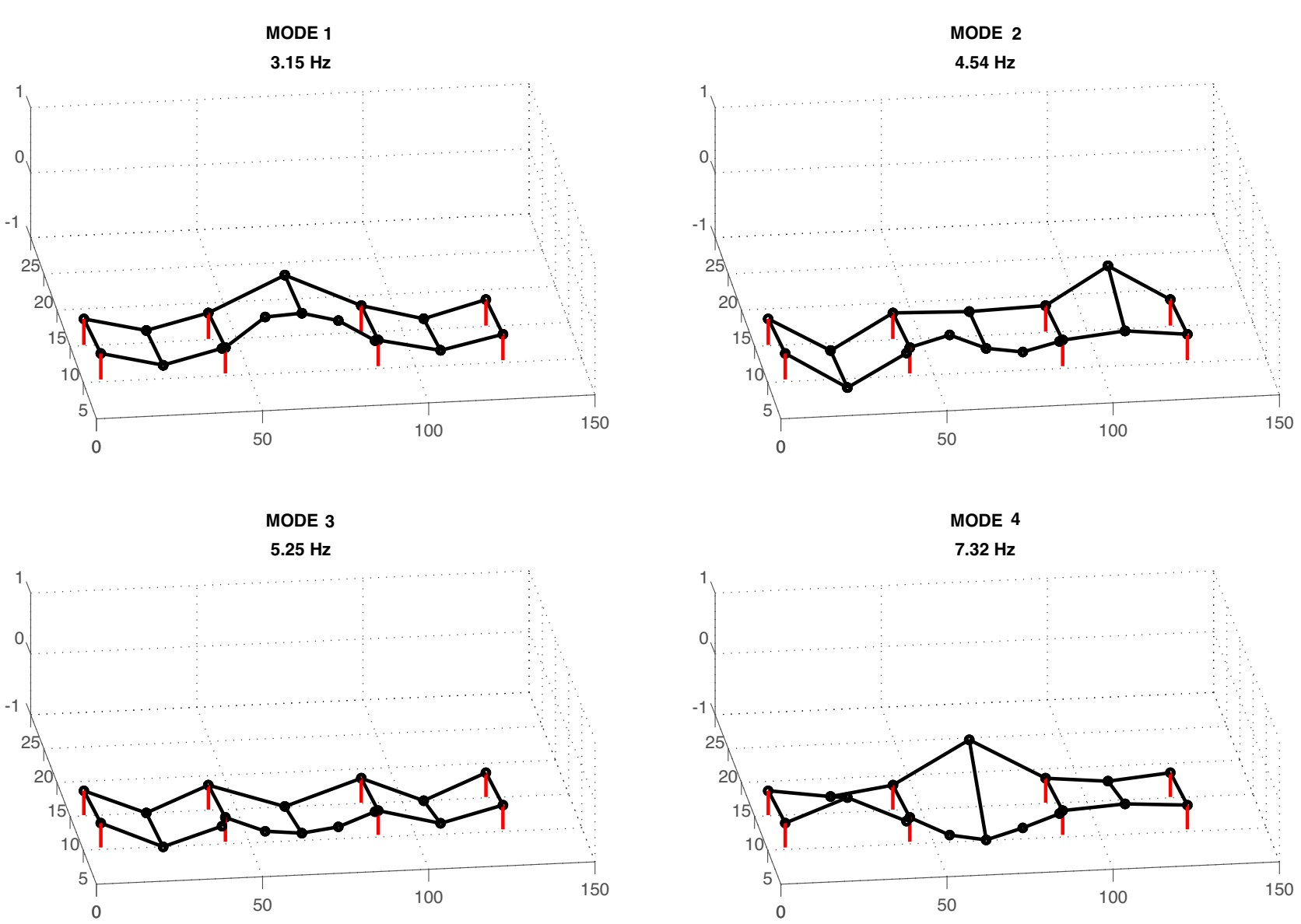

Figure 15. Mode shapes calculated by frequency domain decomposition (FDD) using ambient bridge response data as recorded by the tethered monitoring system (December 2004).

Table 3. Measurement of synchronization time-step delay in the wireless monitoring system.

\begin{tabular}{llllcc}
\hline $\begin{array}{l}\text { Sensor } \\
\text { location }\end{array}$ & $\begin{array}{l}\text { Test 1 } \\
(\mathrm{ms})\end{array}$ & $\begin{array}{l}\text { Test 2 } \\
(\mathrm{ms})\end{array}$ & $\begin{array}{l}\text { Test 3 } \\
(\mathrm{ms})\end{array}$ & $\begin{array}{l}\text { Test 4 } \\
(\mathrm{ms})\end{array}$ & $\begin{array}{l}\text { Test 5 } \\
(\mathrm{ms})\end{array}$ \\
\hline 2 & 0 & 0 & 0 & 0 & 0 \\
4 & 5 & 5 & 0 & 5 & 5 \\
5 & 5 & 5 & 5 & 5 & 5 \\
6 & 5 & 0 & 5 & 5 & 5 \\
7 & 5 & 0 & 5 & 5 & 5 \\
13 & 5 & 0 & 5 & 5 & 5 \\
14 & 5 & 0 & 5 & 5 & 5 \\
15 & 5 & 5 & 5 & 10 & 10 \\
17 & 5 & 5 & 5 & 5 & 5 \\
\hline
\end{tabular}

wireless time-history data with the tethered record. As shown in figure 16 for sensor locations 1 and 2, the error norm exhibits a well defined minimum point. In this case, a one timestep synchronization delay is observed in sensor location 2 compared to sensor location 1 .

The synchronization error is determined for each wireless sensor collocated with a tethered sensor in the July 2005 tests. Table 3 summarizes the synchronization error determined from time-history response data collected during each of the five forced vibration tests. For these tests, the sample rate of both monitoring systems is set at $200 \mathrm{~Hz}$. The wireless monitoring system is accurate in its synchronization with most wireless sensors synchronized within one time step, which corresponds to a synchronization error of $5 \mathrm{~ms}$ or less. Only during the fourth and fifth loading tests is a synchronization error of two time-steps $(10 \mathrm{~ms})$ encountered at sensor 15 . In this study, determination of natural frequencies (as identified by peaks in the Fourier spectra) and mode shapes (as determined by a peak picking method) are unaffected by synchronization errors. However, more rigorous modal analysis methods employed for determination of mode shapes (e.g. FDD) could be negatively impacted by these delays, even when as small as 5-10 ms, since such methods assume precise time synchronization between sensor channels.

\section{Summary and conclusions}

A wireless monitoring system has been proposed to monitor civil structures subjected to ambient and forced vibrations. By using wireless communication between sensors, the costs of wireless monitoring systems are below those of tethered systems that require installation of extensive lengths of coaxial wires. In addition to cost, the performance features of a wireless monitoring system differ greatly from those of tethered counterparts. In particular, wireless monitoring systems are highly decentralized with analogto-digital conversion and data processing performed locally at the wireless sensors, as opposed to at the central data 

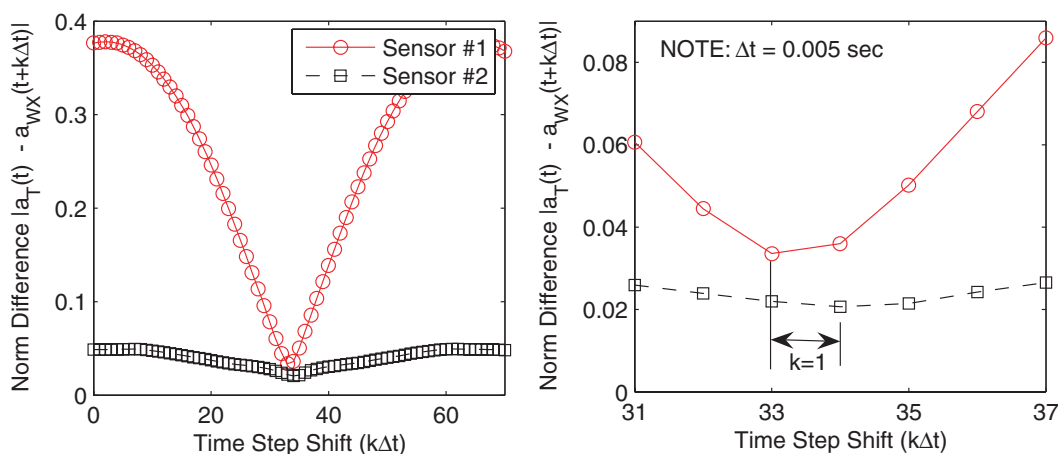

Figure 16. Difference norm between the tethered monitoring system response and the wireless monitoring system recorded response shifted in time (July 2005).

repository. The advantages of embedded data processing include elimination of data glut in the monitoring system, parallel processing of measurement data, and savings in battery power consumed. However, precise time synchronization in a wireless monitoring system remains a challenging task.

To further advance the development of wireless monitoring systems for civil structures, they must be validated in the complex field environment. Prior to this study, field validations of wireless monitoring systems had been limited to systems defined by only a handful of nodes in the monitoring system. In contrast, this study deployed a 14-node wireless monitoring system in the concrete box girder span of the Geumdang Bridge. With accelerometers attached to the wireless sensors, the acceleration response of the bridge was recorded during two sets of forced vibration tests conducted in December 2004 and July 2005. To excite the bridge, trucks of calibrated weight were permitted to transverse the bridge at controlled speeds. During testing in December 2004, the wireless sensors experienced ADC quantization noise that reduced the overall quality of the acceleration response data. To eliminate this noise source, a signal conditioning circuit that amplified the accelerometer outputs was adopted during a second set of field tests conducted in July 2005. As a result of the signal conditioners, the noise in the wireless monitoring system was reduced from 2.2 to $0.13 \mathrm{mg}$. The result was validation of a high-resolution wireless monitoring system whose data quality was on par with that of the tethered system. To synchronize the wireless sensors to a common clock, beacon-based synchronization was attempted. This method was shown to be a robust method of synchronization with synchronization errors bounded by $10 \mathrm{~ms}$ but with most sensors capable of synchronization with errors less than $5 \mathrm{~ms}$.

The wireless monitoring system was shown capable of locally processing measurement data at the sensors. Based upon fast Fourier transform (FFT) and peak picking algorithms, Fourier spectra were calculated by the individual wireless sensors using time-history response data. Once modal frequencies were determined by the wireless sensors, the imaginary components of the Fourier spectra at the modal frequencies were wirelessly exchanged between sensors. These imaginary components were used by the wireless sensors to determine the operational deflection shapes of the bridge. Since the Geumdang Bridge's modal frequencies are well separated and are lightly damped, operational deflection shapes were dominated by the mode shapes of the structure. As such, this distributed computational approach was capable of accurate estimation of mode shapes when the excitation source is broadband.

Future research is still needed to further develop wireless sensors as viable substitutes to traditional tethered sensing technologies. Current work is extending the distributed computing paradigm by embedding additional system identification and damage detection algorithms in the cores of the wireless sensors. This study represents a successful short-term deployment of a wireless monitoring system; future efforts will attempt to deploy a wireless monitoring system for long-term study (on the order of years). To successfully accomplish a long-term deployment, the designer of the monitoring system must explore power sources other than batteries in addition to the pursuit of usage strategies that are more power-efficient. Furthermore, field study is needed to explore the robustness of wireless monitoring systems as the number of nodes increase. As nodal densities grow, there will be a greater need to perform local data processing since the wireless bandwidth will quickly saturate if all wireless sensors try to send their recorded data in real-time.

\section{Acknowledgments}

This research is partially funded by the National Science Foundation under Grants CMS-0421180 and CMS-0528867. YW is supported by a Stanford Graduate Fellowship at Stanford University. Additional support was provided by the KAIST Smart Infra-structure Technology Center (SISTeC), the Korea Highway Corporation and the University of Michigan Rackham Grant and Fellowship Program. The authors would like to express extreme gratitude to Professor Kincho H Law of Stanford University who has provided invaluable expertise in all facets of this research project.

\section{References}

[1] Hipley P 2001 Caltrans' current state-of-practice Proc. Instrumental Systems for Diagnostics of Seismic Response of Bridges and Dams pp 3-7 
[2] Wu Z S 2003 Structural health monitoring and intelligent infrastructures in Japan Proc. 1st Int. Conf. Structural Health Monitoring and Intelligent Infrastructure vol 1, pp 153-67

[3] Ko J M and Ni Y Q 2005 Technology developments in structural health monitoring of large-scale bridges Eng. Struct. 27 1715-25

[4] Celebi M 2002 Seismic Instrumentation of Buildings with Emphasis on Federal Buildings 0-7460-68170 0-7460-68170 (Menlo Park, CA: United States Geological Survey)

[5] Lynch J P and Loh K J 2006 A summary review of wireless sensors and sensor networks for structural health monitoring Shock Vib. Digest 38 91-128

[6] Spencer B F, Ruiz-Sandoval M E and Kurata N 2004 Smart sensing technology: opportunities and challenges J. Struct. Control Health Monitor. 11 349-68

[7] Straser E G and Kiremidjian A S 1998 A modular, wireless damage monitoring system for structures Technical Report no 128 The John A. Blume Earthquake Engineering Center, Stanford, CA

[8] Lynch J P, Law K H, Kiremidjian A S, Carryer E, Farrar C R, Sohn H, Allen D W, Nadler B and Wait J R 2004 Design and performance validation of a wireless sensing unit for structural monitoring applications Struct. Eng. Mech. 17 393-408

[9] Chung H-C, Enotomo T, Loh K and Shinozuka M 2004 Real-time visualization of bridge structural response through wireless MEMS sensors Proc. SPIE-The Int. Society for Optical Engineering 5392 239-46

[10] Ou J P, Li H W, Xiao Y Q and Li Q S 2005 Health dynamic measurement of tall building using wireless sensor network Proc. SPIE-The Int. Society for Optical Engineering $\mathbf{5 7 6 5}$ 205-16

[11] Maxstream 2005 9XCite OEM RF Module Data Sheet

[12] Horowitz P and Hill W 1989 The Art of Electronics (Cambridge: Cambridge University Press)

[13] Wang Y, Lynch J P and Law K H 2006 A wireless structural health monitoring system with multithreaded sensing devices: design and validation Struct. Infrastruct. Eng. at press
[14] Lynch J P, Sundararajan A, Law K H, Kiremidjian A S, Kenny T W and Carryer E 2003 Embedment of structural monitoring algorithms in a wireless sensing unit Struct. Eng. Mech. 15 285-97

[15] Labrosse J J 2002 MicroC OS II: The Real Time Kernel (Gilroy, CA: CMP Books)

[16] Gast M 2005802.11 Wireless Networks (Sebastopol, CA O'Reilly Books)

[17] Lynch J P, Sundararajan A, Law K H, Kiremidjian A S and Carryer E 2004 Embedding damage detection algorithms in a wireless sensing unit for operational power efficiency Smart Mater. Struct. 13 800-10

[18] Brownjohn J M W, Moyo P, Omenzetter P and Lu Y 2003 Assessment of highway bridge upgrading by dynamic testing and finite-element model updating J. Bridge Eng. 8 162-72

[19] Peeters B and Ventura C E 2003 Comparative study of modal analysis techniques for bridge dynamic characteristics Mech. Syst. Signal Process. 17 965-88

[20] Koh H-M, Choo J-F, Kim S and Kil H-B 2005 Applications and researches in bridge health monitoring systems and intelligent infrastructures in Korea Proc. 2nd Int. Conf. on Structural Health Monitoring and Intelligent Infrastructure vol 1, pp 151-62

[21] Lee C G, Lee W T, Yun C B and Choi J S 2004 Development of Integrated System for Smart Evaluation of Load Carrying Capacity of Bridges (Seoul: Korea Highway Corporation)

[22] PCB-Piezotronics 2005393 B12 ICP Seismic Accelerometer Data Sheet

[23] PCB-Piezotronics 2005 3801D1FB3G Capacitive Accelerometer Data Sheet

[24] Brincker R, Zhang L and Andersen P 2001 Modal identification of output-only systems using frequency domain decomposition Smart Mater. Struct. 10 441-5

[25] Farrar C R and James G H 1997 System identification from ambient vibration measurements on a bridge J. Sound Vib. $2051-18$

[26] Lei Y, Kiremidjian A S, Nair K K, Lynch J P and Law K H 2005 Algorithms for time synchronization of wireless structural monitoring sensors Earthq. Eng. Struct. Dyn. 34 555-73 